CARNETS DE Carnets de géographes

GÉOGRAPHES.

$14 \mid 2020$

Varia

\title{
Gestion du paysage en faveur du réseau écologique
}

Modes de gouvernance, représentations et pratiques dans les espaces agricoles

Julie Delclaux

\section{OpenEdition}

Journals

Édition électronique

URL : https://journals.openedition.org/cdg/6261

DOI : $10.4000 / \mathrm{cdg} .6261$

ISSN : 2107-7266

Éditeur

UMR 245 - CESSMA

Référence électronique

Julie Delclaux, "Gestion du paysage en faveur du réseau écologique », Carnets de géographes [En

ligne], 14 | 2020, mis en ligne le 30 novembre 2020, consulté le 22 mai 2021. URL : http://

journals.openedition.org/cdg/6261; DOI : https://doi.org/10.4000/cdg.6261

Ce document a été généré automatiquement le 22 mai 2021.

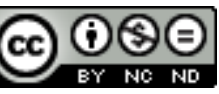

La revue Carnets de géographes est mise à disposition selon les termes de la Licence Creative Commons Attribution - Pas d'Utilisation Commerciale - Pas de Modification 4.0 International. 


\title{
Gestion du paysage en faveur du réseau écologique
}

\author{
Modes de gouvernance, représentations et pratiques dans les espaces \\ agricoles
}

Julie Delclaux

1 Les politiques de conservation de la biodiversité ne concernent plus seulement aujourd'hui des espaces protégés, mais également des réseaux où l'enjeu est de préserver et restaurer les continuités entre habitats des espèces afin de favoriser la circulation de la faune. En France, cette approche se traduit depuis 2007 par la politique Trame verte et bleue. Ce nouveau regard élargit les territoires de la conservation aux espaces de nature ordinaire, tels que les espaces agricoles, où les processus écologiques et les activités humaines sont fortement imbriqués. Si les espaces agricoles apparaissent souvent comme des lieux de conflit entre «nature » et agriculture, entre objectifs de production et de conservation, les logiques basées sur le réseau et les fonctionnalités écologiques du paysage réinterrogent ces oppositions.

2 Mon travail de thèse s'inscrit dans ce contexte et questionne une conservation intégrée de la biodiversité dans les espaces agricoles : en quoi et dans quelle mesure la mise en œuvre d'une gestion locale du paysage en faveur de ses fonctionnalités écologiques permettrait-elle une meilleure synergie entre production agricole et conservation de la biodiversité ? Le paysage peut se définir comme la " partie de territoire telle que perçue par les populations, dont le caractère résulte de l'action de facteurs naturels et/ou humains et de leurs interrelations » (Convention européenne du paysage, 2000). En ce sens, je considère le paysage comme un système socio-écologique afin d'approcher la complexité des interactions entre ces deux dimensions, et je mobilise le cadre d'analyse de la durabilité des systèmes socio-écologiques. Ce cadre, développé par Ostrom et ses collègues, questionne la capacité des acteurs à proposer une gestion commune durable d'une ressource. Il décompose le système socio-écologique étudié en différents sous-système relatifs à la ressource elle-même, à la gouvernance et aux acteurs impliqués, et propose une liste de variables à caractériser pour décrire le système. Le paysage et ses fonctionnalités écologiques constituent, dans cette thèse, la ressource étudiée. Ma 
posture de géographe de la conservation positionne ce travail au carrefour de la géographie, de la géoagronomie, de l'économie institutionnelle et de la sociologie des organisations. J'observe ainsi en particulier les modes de gouvernance, les représentations et les pratiques agricoles.

3 L'originalité de mon travail est de déployer une approche associant méthodes d'analyse qualitative et quantitative à différentes échelles afin de proposer un regard complexe et systémique. À l'échelle nationale et régionale, ce sont les évolutions des représentations sociétales des relations entre agriculture et biodiversité qui sont analysées au moyen d'une analyse de contenu d'articles de la presse quotidienne française sur près de vingt ans. Le corpus de presse nationale (2 547 articles) et celui de presse régionale (1344 articles) sont explorés grâce à des outils de la statistique textuelle. À l'échelle de l'ancienne région Rhône-Alpes, ce sont la gouvernance et la mise en œuvre d'un outil contractuel de la politique Trame verte et bleue qui sont comparées dans le cadre de trois études de cas (le pays de Bièvre-Valloire en Isère, le SCoT du Grand Rovaltain dans la Drôme et la Communauté d'Agglomération LoireForez dans la Loire). Le choix de ces terrains d'étude, effectué en collaboration avec les acteurs locaux dans un projet de recherche-action, s'est appuyé sur plusieurs critères : présence d'outils territoriaux de prise en compte des continuités écologiques, agriculture intensive et paysage de plaine. Pour cela, la démarche associe entretiens semi-directifs ( 23 entretiens avec les acteurs locaux) et observations participantes de réunions de mise en œuvre de cette politique publique. Enfin et à l'échelle d'exploitations agricoles localisées dans les trois cas comparés, des entretiens semidirectifs conduits auprès de 25 agriculteurs ont permis d'identifier l'importance et les modalités d'intégration de la biodiversité comme facteur de décision des pratiques des agriculteurs ainsi que leurs représentations de la biodiversité et des éléments seminaturels arborés tels que les haies.

4 Les résultats obtenus mettent en évidence que le paysage est un outil propice à l'insertion de la biodiversité dans les espaces agricoles, et à celle des acteurs locaux dans une stratégie de conservation. Il permet en effet de mettre en relation des mondes sociaux hétérogènes et constitue une source de transversalité entre les politiques publiques environnementales des territoires. Cependant, la notion de paysage qui fait référence dans la politique Trame verte et bleue reste largement celle de l'écologie et celle-ci peine à inclure la dimension agricole et les agriculteurs. Ainsi, la gestion du paysage en faveur de ses fonctionnalités écologiques ne parvient pas encore à une double intégration: celle de l'hétérogénéité spatiale et sociale des espaces agricoles dans la conservation de la biodiversité d'une part, et celle de la conservation de la biodiversité dans l'activité productive agricole d'autre part. Enfin, les apports théoriques et méthodologiques de ce travail concernent l'articulation d'échelles et de thématiques. En effet, pour porter un regard géographique composant à la fois avec les dimensions sociologique, politique, économique et naturaliste, je mobilise plusieurs objets d'étude, données et méthodes à différentes échelles spatiale et temporelle. Ce travail révèle que la mise en œuvre d'une géographie sociale et écologique reste une perspective scientifique à poursuivre. 
Figure 1. Plaine du Rovaltain dans la Drôme vue depuis les contreforts du Vercors

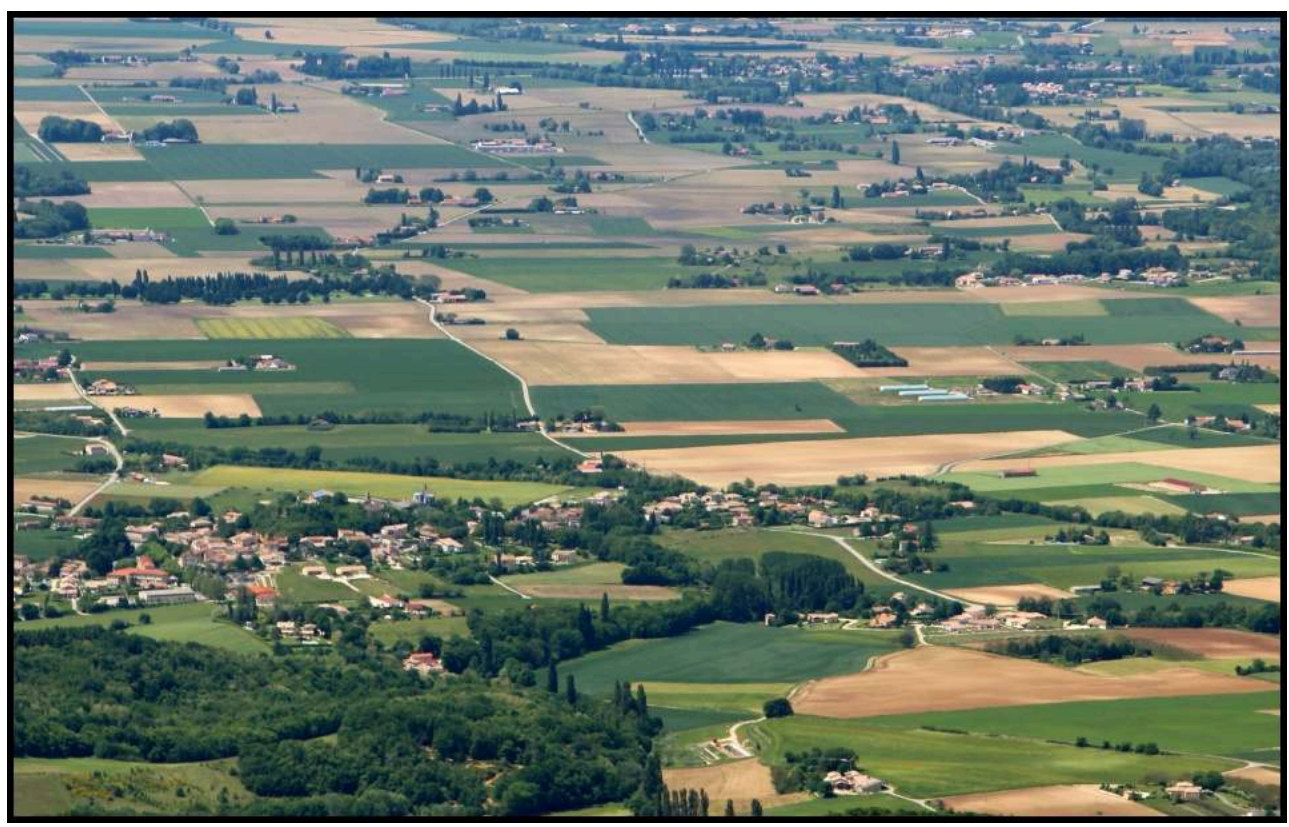

Dans les espaces agricoles, pour comprendre et faire du réseau écologique un objet de politique publique, il convient de prendre en compte ses dimensions sociales et pas seulement écologiques.

\author{
Discipline \\ Géographie

\section{Directeur} \\ Philippe Fleury, ISARA / Laboratoire d'Etudes Rurales

\section{Université} \\ Université Lyon Lumière 2
}

Membres du jury de thèse, soutenue le 24 septembre 2020

Philippe FLEURY, Maître de Conférences HDR, ISARA / Laboratoire d'Etudes Rurales (directeur)

Sylvie GUILLERME, Chargée de recherche HDR, Laboratoire Géode, CNRS Pascal MARTY, Professeur, LADYSS, Université Paris I Panthéon-Sorbonne Raphaël MATHEVET, Directeur de recherche, CNRS/Institut français de Pondichéry (rapporteur)

Patrick MOQUAY, Professeur directeur délégué à la recherche, ENSP Versailles Julie RUIZ, Professeure, Université du Québec à Trois Rivières (rapportrice) Sylvie VANPEENE-BRUHIER, Ingénieure de recherche ICPEF, INRAE Centre PACA

\title{
Situation professionnelle à l'issue de la thèse
}

En recherche de post-doc

\section{Courriel de l'auteur}

delclaux.julie[at]gmail.com 
INDEX

Thèmes : Carnets de soutenances 